\title{
OPEN Dyschloremia is associated with failure to restore renal function in survivors with acute kidney injury: an observation retrospective study
}

\begin{abstract}
Youn Kyung Kee, Hee Jung Jeon, Jieun Oh \& Dong Ho Shin
Dyschloremia is common in critically ill patients. However, little is known about the effects of dyschloremia on renal function in patients with acute kidney injury (AKI) requiring continuous renal replacement therapy (CRRT). A total of 483 patients who received CRRT for AKI were selected and divided into three groups according to their serum chloride concentrations at the time of CRRT initiation. At 90 days after initiating CRRT, renal outcome, i.e., non-complete renal recovery, or renal failure, was assessed in the three groups. The hypochloremia group (serum chloride concentrations $<96 \mathrm{mEq} / \mathrm{L}, \mathrm{n}=60$ ), the normochloremia group (serum chloride concentrations, 96-111 mEq/L, $\mathrm{n}=345$ ), and the hyperchloremia group (serum chloride concentrations $>111 \mathrm{mEq} / \mathrm{L}$, $\mathrm{n}=78$ ) were classified. The simplified acute physiology score III was higher in the hyperchloremia and hypochloremia groups than in the normochloremia group. Multivariate logistic regression analyses showed that hypochloremia (odds ratio, 5.12; 95\% confidence interval $[\mathrm{Cl}], 2.56-10.23 ; P<0.001$ ) and hyperchloremia (odds ratio, $2.53 ; 95 \% \mathrm{Cl}, 1.25-5.13 ; P=0.01$ ) were significantly associated with non-complete renal recovery. Similar trends were observed for renal failure. This study showed that dyschloremia was independently associated with failure in restoring renal function following AKI.
\end{abstract}

Severe acute kidney injury (AKI) requiring renal replacement therapy (RRT) is a common serious complication in critically ill patients and is associated with high mortality and morbidity ${ }^{1-4}$. Although critical care and dialysis technology have improved significantly, the mortality in patients with severe AKI requiring RRT is higher than in those with other serious diseases, such as acute respiratory distress syndrome or myocardial infarction ${ }^{5,6}$. In addition, survivors with severe AKI requiring RRT are known to have a high risk of developing long-term complications, such as chronic kidney disease (CKD) or renal failure ${ }^{4}$.

Chloride is a major extracellular anion in the blood which constitutes approximately one-third of extracellular fluid tonicity ${ }^{7,8}$. In addition, it plays several important roles in the body, such as maintaining electrical activity, acid-base balance, fluid and gastrointestinal homeostasis, and renal function ${ }^{7,9-11}$. Despite its physiological and clinical importance, less attention has been paid to chloride than other routinely measured electrolytes in critically ill patients ${ }^{8}$. Dyschloremia commonly observed in critically ill patients can occur because of various etiologic factors associated with the illness or treatment ${ }^{7,12,13}$. Although there have been few outcome-related studies on dyschloremia, several studies showed that dyschloremia is associated with significantly increased mortality and morbidity ${ }^{11,14-16}$. Some studies have shown that hyperchloremia could reduce renal blood flow and glomerular filtration rate (GFR) and consequently, cause salt and water retention to occur ${ }^{17,18}$. These findings suggest that hyperchloremia is associated with AKI based on clear biological plausibility. In fact, accumulation of clinical evidence also suggests that hyperchloremia is associated with AKI in critically ill patients ${ }^{19,20}$. Although there is no clear experimental evidence for hypochloremia-associated AKI, some observational clinical studies have shown that hypochloremia is associated with $\mathrm{AKI}^{21,22}$. Moreover, renal recovery following AKI is clinically important because AKI had been found to be an independent risk factor for the development of CKD or the progression from CKD to renal failure ${ }^{23}$. However, there is limited knowledge regarding the association between serum chloride concentrations and renal recovery in patients with AKI. Thus, this study aimed to determine 


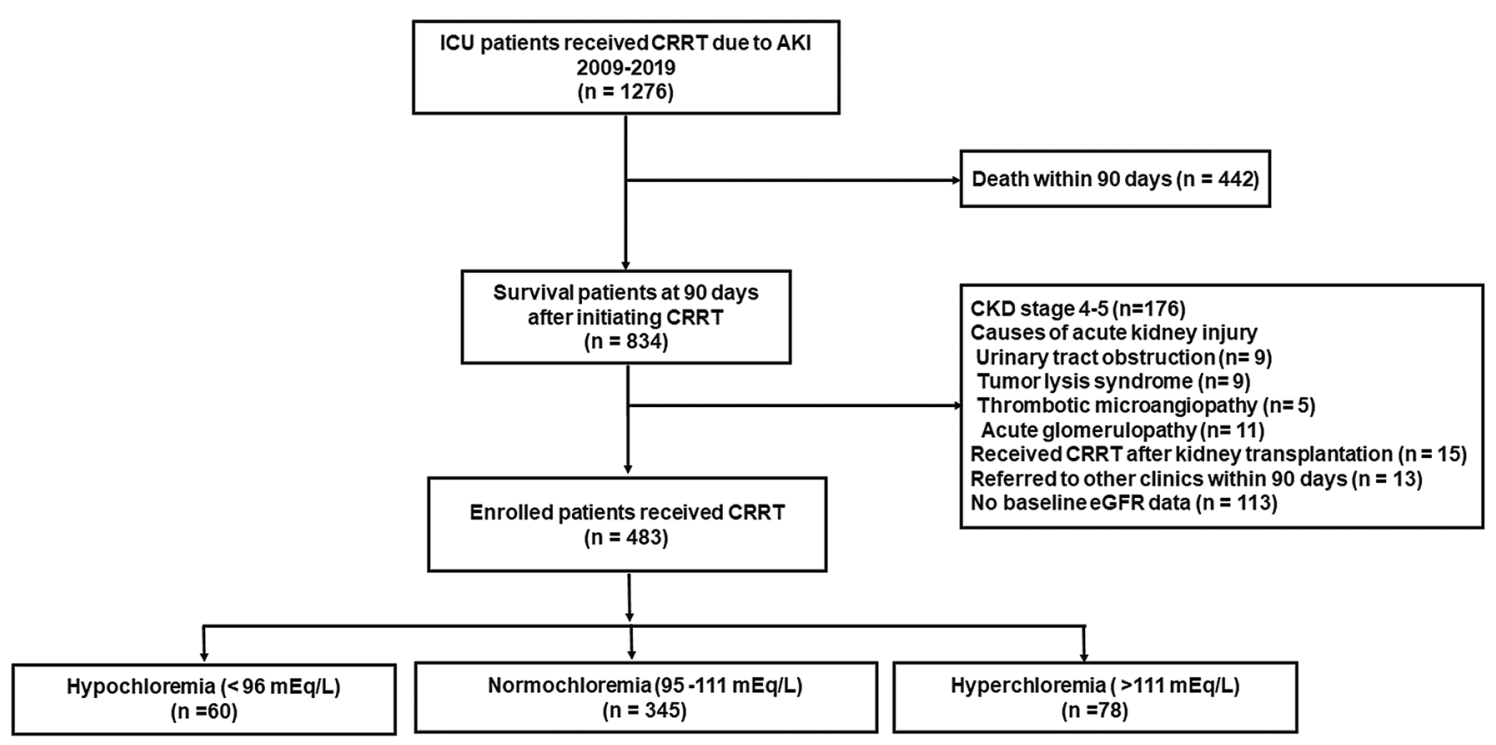

Figure 1. Patient inclusions and subgroupings.

whether dyschloremia was associated with failure to restore renal function in survivors having severe AKI and requiring RRT.

\section{Results}

Study population. During the 10-year study period, 1276 patients were included. Of 1276 patients, 834 patients survived at 90 days after initiating continuous renal replacement therapy (CRRT). Of 834 survivors, 351 were excluded: 176 patients with severe CKD, 34 patients whose cause of AKI was urinary tract obstruction, tumor lysis syndrome, thrombotic microangiopathy or acute glomerulopathy, 15 patients who received CRRT after kidney transplantation, 13 patients who were referred to other clinics within 90 days, and 113 patients without records in the laboratory database within 12 months before hospital admission (Fig. 1).

Characteristics at the time of CRRT. Overall, 483 patients with AKI requiring CRRT were included in this study, of whom 293 were men. The mean age of the patients was 65.6 years, and sepsis in 154 patients (31.9\%) was the most common cause of AKI at the time of CRRT initiation. The median Charlson Comorbidity Index (CCI), Sequential Organ Failure Assessment (SOFA) score, and mean simplified acute physiology score III (SAPS III) were $2.0(0.0-3.0), 9$ (8.0-11.0), and 43.9 \pm 8.5 , respectively. The patients were divided into three groups according to the degree of dyschloremia (hypochloremia, normochloremia, and hyperchloremia); 60 $(12.4 \%)$ were in the hypochloremia group, $345(71.4 \%)$ were in the normochloremia group, and $78(16.1 \%)$ were in the hyperchloremia group. Although patients with hypochloremia had comparable CCIs to patients with normochloremia, those with hypochloremia had a higher prevalence of congestive heart failure than those with normochloremia. Meanwhile, patients with hypochloremia and hyperchloremia had higher SAPS III than patients with normochloremia (Table 1). Of note, all patients had blood sampling at 90 days after CRRT initiation.

Kidney outcome according to chloride category. Of 483 patients, 111 (23\%) had non-complete renal recovery and $69(14.3 \%)$ had renal failure at 90 days after initiating CRRT. The incidence of non-complete renal recovery was significantly lower in the normochloremia group than in the other groups $(P<0.001)$. Although there was no significant difference in the incidence of renal failure among three groups, a similar pattern was observed in the development of renal failure (Fig. 2).

Factors associated with renal outcome. In logistic regression analysis, CKD, CHF, high CCIs, high creatinine concentrations, and dyschloremia were independently associated with non-complete renal recovery. Specifically, multivariate logistic regression analysis showed that hypochloremia and hyperchloremia were significantly associated with a $>$ fivefold (odds ratio, 5.12; 95\% confidence interval $[\mathrm{CI}], 2.56-10.23 ; P<0.001$ ) and a twofold (odds ratio, 2.53; 95\% CI, 1.25-5.13; $P=0.01$ ) higher risk of non-complete renal recovery, respectively (Table 2). Meanwhile, dyschloremia was also independently associated with the development of renal failure. However, only hypochloremia was significantly associated with a > twofold (odds ratio, 2.74; 95\% CI, 1.19-6.32, $P=0.02$ ) higher risk of renal failure (Table 3 ).

Association between serum chloride concentration as a continuous variable and renal outcome. Predicted odds ratios of renal outcome according to continuous values of serum chloride concentrations were generated using a cubic spline relationship. The graph shows a bidirectional or U-shaped association between serum chloride concentrations and non-complete renal recovery in the multivariate logistic regression model. In addition, when renal failure was considered as a renal outcome, a similar pattern was observed (Fig. 3). 


\begin{tabular}{|c|c|c|c|c|}
\hline Variable & Hypochloremia $(n=60)$ & Normochloremia $(n=345)$ & Hyperchloremia $(n=78)$ & $P$-value \\
\hline \multicolumn{5}{|l|}{ Demographic characteristics } \\
\hline Age (years) & $63.5(57.0-72.0)$ & $66.0(55.0-75.0)$ & $67.5(55.0-79.0)$ & 0.23 \\
\hline Male, n (\%) & $37(61.7)$ & $212(61.4)$ & $44(56.4)$ & 0.70 \\
\hline \multicolumn{5}{|l|}{ Comorbid disease } \\
\hline CKD, n (\%) & $11(18.3)$ & $42(12.2)$ & $7(9.0)$ & 0.25 \\
\hline MI, n (\%) & $5(8.3)$ & $39(11.3)$ & $8(10.3)$ & 0.78 \\
\hline CHF, n (\%) & $20(33.3)$ & $48(13.9)$ & $8(10.3)$ & $<0.001$ \\
\hline CVA, n (\%) & $6(10.0)$ & $59(17.1)$ & $12(15.4)$ & 0.38 \\
\hline PVD, n (\%) & $3(5.0)$ & $16(4.6)$ & $4(5.1)$ & 0.98 \\
\hline Dementia, n (\%) & $6(10)$ & $21(6.1)$ & $5(6.4)$ & 0.53 \\
\hline $\mathrm{DM}, \mathrm{n}(\%)$ & $7(11.7)$ & $27(7.8)$ & $7(9.0)$ & 0.61 \\
\hline Cirrhosis, n (\%) & $4(6.7)$ & $19(5.5)$ & $2(2.6)$ & 0.49 \\
\hline COPD, n (\%) & $2(3.3)$ & $10(2.9)$ & $4(5.1)$ & 0.61 \\
\hline Malignancy, n (\%) & $6(10.0)$ & $36(10.4)$ & $5(6.4)$ & 0.56 \\
\hline Charlson comorbidity index & $2.0(0.0-4.0)$ & $2(0-3.0)^{* *}$ & $1.0(0.0-2.0)^{\#}$ & 0.02 \\
\hline \multicolumn{5}{|l|}{ Cause of AKI requiring RRT } \\
\hline Sepsis, n (\%) & $18(30.0)$ & $103(29.9)$ & $33(42.3)$ & 0.10 \\
\hline Ischemia, n (\%) & $14(23.0)$ & $93(27.0)$ & $16(20.5)$ & 0.46 \\
\hline Surgery, n (\%) & $3(5.0)$ & $47(13.6)$ & $5(6.4)$ & 0.06 \\
\hline Others, n (\%) & 25 (41.7) & $102(29.6)$ & $24(30.8)$ & 0.17 \\
\hline \multicolumn{5}{|l|}{ Clinical data } \\
\hline Time to start CRRT (days) & $1.0(0.8-1.3)$ & $1.1(0.8-1.5)$ & $1.1(0.7-1.7)$ & 0.27 \\
\hline SOFA score & $9.0(8.1-11.0)$ & $9.0(8.0-11.0)$ & $10.0(8.0-12.0)$ & 0.158 \\
\hline SAPS III & $48.9 \pm 6.4^{*}$ & $42.6 \pm 8.5^{* *}$ & $45.8 \pm 8.3^{\#}$ & $<0.001$ \\
\hline Urine output $(\mathrm{mL} / \mathrm{h})$ & $7(5-8)$ & $6(5-8)$ & $6(5-8)$ & 0.229 \\
\hline Loop diuretic therapy (\%) & $55(91.7)$ & $309(89.6)$ & $63(80.8)$ & 0.06 \\
\hline Fluid balance (L/day) & $1.8(1.2-2.0)$ & $1.9(1.5-2.1)$ & $1.8(1.4-2.2)$ & 0.088 \\
\hline Chloride administration (mEq/day) & $295.3(215.0-340.3)$ & $317.2(263.0-373.0)$ & $321.9(232.8-370.5)$ & 0.07 \\
\hline \multicolumn{5}{|l|}{ Biochemical data } \\
\hline $\mathrm{WBC}\left(10^{3} / \mathrm{mm}^{3}\right)$ & $10.2(6.9-16.1)$ & $11.3(7.7-16.6)$ & $10.9(8.0-17.9)$ & 0.69 \\
\hline Hct (\%) & $28.0(23.8-33.5)$ & $28.4(24.0-32.8)$ & $29.1(22.5-34.8)$ & 0.80 \\
\hline Platelet $\left(10^{3} / \mathrm{mm}^{3}\right)$ & $159.0(91.0-233.0)$ & $123.0(68.0-200.0)$ & $123.5(77.0-187.0)$ & 0.09 \\
\hline $\mathrm{BUN}(\mathrm{mg} / \mathrm{dL})$ & $52.5(20.2-79.8)$ & $49.1(29.3-72.4)$ & $46.3(25.6-67.1)$ & 0.75 \\
\hline Baseline $\mathrm{Cr}(\mathrm{mg} / \mathrm{dL})$ & $1.1(0.8-1.3)$ & $1.0(0.8-1.3)$ & $1.1(0.9-1.3)$ & 0.08 \\
\hline Baseline eGFR $\left(\mathrm{mL} / \mathrm{min}\right.$ per $\left.1.73 \mathrm{~m}^{2}\right)$ & $65.2(62.2-69.1)$ & $67.2(63.3-81.0)$ & $66.5(63.2-72.7)$ & 0.26 \\
\hline $\operatorname{Cr}(\mathrm{mg} / \mathrm{dL})$ & $5.2(3.2-8.5)^{\star}$ & $4.2(2.6-6.6)^{\star \star}$ & $3.0(2.1-4.7)^{\#}$ & $<0.001$ \\
\hline eGFR $\left(\mathrm{mL} / \mathrm{min}\right.$ per $\left.1.73 \mathrm{~m}^{2}\right)$ & $9.5(5.6-15.8)^{*}$ & $12.9(7.5-23.4)^{* *}$ & $19.2(11.2-28.0)^{\#}$ & $<0.001$ \\
\hline $\mathrm{Na}(\mathrm{mEq} / \mathrm{L})$ & $133(129-138)^{*}$ & $137(134-141)^{* *}$ & $144(140-149)^{\#}$ & $<0.001$ \\
\hline $\mathrm{K}(\mathrm{mEq} / \mathrm{L})$ & $4.5(4.1-5.5)$ & $4.4(3.8-5.2)$ & $4.4(3.7-5.1)$ & 0.38 \\
\hline $\mathrm{TCO}_{2}(\mathrm{mmol} / \mathrm{L})$ & $17.4 \pm 6.5$ & $17.7 \pm 5.0^{* *}$ & $14.8 \pm 4.6^{\#}$ & $<0.001$ \\
\hline Total bilirubin (mg/dL) & $0.5(0.4-1.1)$ & $0.7(0.5-1.3)$ & $0.7(0.5-1.2)$ & 0.06 \\
\hline
\end{tabular}

Table 1. Patients' characteristics according to chloride category. CKD, chronic kidney disease; MI, myocardial infarction; $\mathrm{CHF}$, congestive heart failure; CVA, cerebrovascular accident; PVD, peripheral vascular disease; COPD, chronic obstructive pulmonary disease; AKI, acute kidney injury; CRRT, continuous renal replacement therapy; SOFA, Sequential Organ Failure Assessment; SAPS III, Simplified Acute Physiology Score III; CCI, Charlson comorbidity index; SBP, systolic blood pressure; DBP, diastolic blood pressure; MAP, mean arterial pressure; WBC, white blood cell; Hct, hematocrit; Cr, creatinine; BUN, blood urea nitrogen; eGFR, estimated glomerular filtration rate; $\mathrm{Na}$, sodium; $\mathrm{K}$, potassium; $\mathrm{TCO}_{2}$, total carbon dioxide. Categorical variables were compared by chi-square test or Fisher's exact test. Continuous variables were compared by Analysis of variance (AVONVA) or the Kruskal-Wallis test. Of note, Bonferonni post hoc tests or Dunn procedure was used for pairwise comparisons. ${ }^{*}, P<0.05$ versus normochloremia group; ${ }^{*}, P<0.05$ versus hyperchloremia group; $\#, P<0.05$ versus hypochloremia group. Values have been expressed as means \pm standard deviations or as numbers (percentages). 

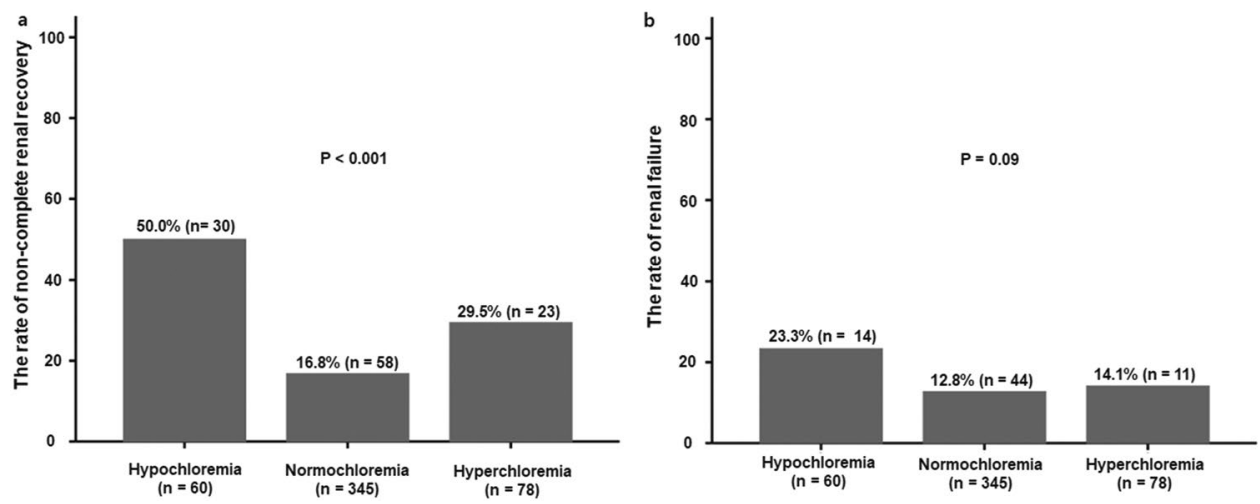

Figure 2. The rate of failure to restore renal function. (a) The incidence of non-complete renal recovery was significantly lower in the normochloremia group than in the other groups $(P<0.001)$. (b) There was no significant difference in the incidence of renal failure among three groups $(P=0.09)$. Categorical variables were compared by chi-square test or Fisher's exact test.

\begin{tabular}{|c|c|c|c|c|}
\hline \multirow[b]{2}{*}{ Variable } & \multicolumn{2}{|c|}{ Univariate analysis } & \multicolumn{2}{|c|}{ Multivariate analysis $^{\mathrm{a}}$} \\
\hline & OR $(95 \% \mathrm{CI})$ & $P$-value & OR $(95 \% \mathrm{CI})$ & $P$-value \\
\hline Age (per 1 year) & $1.01(0.99-1.02)$ & 0.35 & $1.01(0.99-1.03)$ & 0.23 \\
\hline Male (vs. Female) & $0.70(0.46-1.08)$ & 0.11 & $0.66(0.41-1.08)$ & 0.10 \\
\hline \multicolumn{5}{|c|}{ Risk factors for deteriorating kidney function } \\
\hline CKD & $2.80(1.59-4.93)$ & $<0.001$ & $2.12(1.10-4.08)$ & 0.02 \\
\hline CHF & $2.12(1.25-3.59)$ & 0.01 & $1.36(0.71-2.59)$ & 0.36 \\
\hline CCI & $1.18(1.07-1.29)$ & 0.001 & $1.15(1.02-1.28)$ & 0.02 \\
\hline \multicolumn{5}{|l|}{ Cause of AKI required CRRT } \\
\hline Sepsis & $1.21(0.77-1.89)$ & 0.40 & & \\
\hline Surgery & $0.93(0.47-1.83)$ & 0.83 & & \\
\hline Ischemia & $0.87(0.53-1.43)$ & 0.57 & & \\
\hline Other & $0.96(0.61-1.52)$ & 0.87 & & \\
\hline \multicolumn{5}{|l|}{ Clinical data } \\
\hline SOFA score & $1.08(0.90-1.19)$ & 0.09 & $1.10(0.99-1.22)$ & 0.08 \\
\hline SAPS III & $1.02(0.99-1.05)$ & 0.08 & $0.99(0.95-1.03)$ & 0.56 \\
\hline Urine output (per $1 \mathrm{~mL} / \mathrm{h}$ ) & $1.05(0.98-1.12)$ & 0.16 & $1.06(0.98-1.14)$ & 0.15 \\
\hline $\mathrm{Cr}($ per $1 \mathrm{mg} / \mathrm{dL})$ & $1.11(1.04-1.18)$ & 0.001 & $1.11(1.02-1.20)$ & 0.01 \\
\hline $\mathrm{Na}($ per $1 \mathrm{mEq} / \mathrm{L})$ & $0.99(0.98-1.02)$ & 0.76 & $1.01(0.97-1.04)$ & 0.79 \\
\hline $\mathrm{K}($ per $1 \mathrm{mEq} / \mathrm{L})$ & $0.98(0.81-1.20)$ & 0.87 & $0.78(0.61-0.99)$ & 0.04 \\
\hline $\mathrm{TCO}_{2}($ per $1 \mathrm{mmol} / \mathrm{L})$ & $0.98(0.94-1.02)$ & 0.26 & $0.97(0.93-1.02)$ & 0.24 \\
\hline Total bilirubin (per $1 \mathrm{mg} / \mathrm{dL})$ & $0.96(0.89-1.04)$ & 0.35 & $0.99(0.92-1.07)$ & 0.84 \\
\hline \multicolumn{5}{|l|}{ Dyschloremia } \\
\hline Normochloremia & Reference & & Reference & \\
\hline Hyperchloremia & $2.07(1.18-3.63)$ & 0.01 & $2.53(1.25-5.13)$ & 0.01 \\
\hline Hypochloremia & $4.95(2.77-8.83)$ & $<0.001$ & $5.12(2.56-10.23)$ & $<0.001$ \\
\hline
\end{tabular}

Table 2. Factors associated with non-complete renal recovery. CKD, chronic kidney disease; CHF, congestive heart failure; CCI, Charlson comorbidity index; SAPS III, Simplified Acute Physiology Score III; SOFA, Sequential Organ Failure Assessment; CRRT, continuous renal replacement therapy; AKI, acute kidney injury; $\mathrm{Cr}$, creatinine; $\mathrm{Na}$, sodium; $\mathrm{K}$, potassium; $\mathrm{TCO}_{2}$, total carbon dioxide; $\mathrm{OR}$, odds ratio; $\mathrm{CI}$, confidence interval. ${ }^{a}$ Adjusted for age, male, CKD, CHF, CCI, SAPS III score, SOFA, urine output, $\mathrm{Cr}, \mathrm{Na}, \mathrm{K}, \mathrm{TCO}_{2}$, total bilirubin, hyperchloremia, and hypochloremia.

\section{Discussion}

This retrospective study showed that dyschloremia was independently associated with failure to restore renal function in survivors with severe AKI requiring RRT. Notably, when serum chloride concentrations were considered as continuous values, there was a U-shaped association between serum chloride concentrations and failure to restore renal function. 


\begin{tabular}{|c|c|c|c|c|}
\hline \multirow[b]{2}{*}{ Variable } & \multicolumn{2}{|c|}{ Univariate analysis } & \multicolumn{2}{|c|}{ Multivariate analysis $^{\mathrm{a}}$} \\
\hline & OR $(95 \% \mathrm{CI})$ & $P$-value & OR $(95 \% \mathrm{CI})$ & $P$-value \\
\hline Age (per 1 year) & $1.01(0.99-1.02)$ & 0.64 & $1.03(1.00-1.05)$ & 0.05 \\
\hline Male (vs. Female) & $0.94(0.56-1.58)$ & 0.82 & $0.99(0.55-1.76)$ & 0.96 \\
\hline \multicolumn{5}{|c|}{ Risk factors for deteriorating kidney function } \\
\hline CKD & $2.54(1.34-4.82)$ & 0.004 & $1.60(0.78-3.30)$ & 0.20 \\
\hline $\mathrm{CHF}$ & $2.38(1.31-4.33)$ & 0.04 & $1.83(0.88-3.78)$ & 0.11 \\
\hline CCI & $1.20(1.07-1.33)$ & 0.001 & $1.18(1.04-1.35)$ & 0.01 \\
\hline \multicolumn{5}{|l|}{ Cause of AKI required RRT } \\
\hline Sepsis & $0.85(0.49-1.49)$ & 0.58 & & \\
\hline Surgery & $1.20(0.56-2.58)$ & 0.64 & & \\
\hline Ischemia & $0.95(0.53-1.71)$ & 0.87 & & \\
\hline Other & $1.12(0.65-1.92)$ & 0.69 & & \\
\hline SAPS III & $0.98(0.96-1.02)$ & 0.34 & $1.01(0.95-1.04)$ & 0.35 \\
\hline SOFA score & $1.11(0.99-1.24)$ & 0.07 & $1.14(1.00-1.31)$ & 0.05 \\
\hline \multicolumn{5}{|l|}{ Clinical data } \\
\hline Urine output (per $1 \mathrm{~mL} / \mathrm{h}$ ) & $1.02(0.94-1.10)$ & 0.66 & $1.01(0.92-1.10)$ & 0.91 \\
\hline $\mathrm{Cr}($ per $1 \mathrm{mg} / \mathrm{dL})$ & $1.15(1.07-1.23)$ & $<0.001$ & $1.11(1.02-1.21)$ & 0.02 \\
\hline $\mathrm{Na}($ per $1 \mathrm{mEq} / \mathrm{L})$ & $0.99(0.97-1.02)$ & 0.81 & $1.02(0.97-1.06)$ & 0.48 \\
\hline $\mathrm{K}($ per $1 \mathrm{mEq} / \mathrm{L})$ & $1.04(0.83-1.32)$ & 0.72 & $0.79(0.59-1.03)$ & 0.08 \\
\hline $\mathrm{TCO}_{2}($ per $1 \mathrm{mmol} / \mathrm{L})$ & $0.95(0.91-1.00)$ & 0.06 & $0.93(0.88-0.99)$ & 0.01 \\
\hline Total bilirubin (per $1 \mathrm{mg} / \mathrm{dL}$ ) & $0.93(0.82-1.05)$ & 0.25 & $0.99(0.89-1.09)$ & 0.79 \\
\hline \multicolumn{5}{|l|}{ Dyschloremia } \\
\hline Normochloremia & Reference & & Reference & \\
\hline Hyperchloremia & $1.12(0.55-2.29)$ & 0.75 & $1.26(0.53-3.01)$ & 0.60 \\
\hline Hypochloremia & $2.08(1.05-4.10)$ & 0.03 & $2.74(1.19-6.32)$ & 0.02 \\
\hline
\end{tabular}

Table 3. Factors associated with renal failure. CKD, chronic kidney disease; CHF, congestive heart failure; CCI, Charlson comorbidity index; SAPS III, Simplified Acute Physiology Score III; SOFA, Sequential Organ Failure Assessment; CRRT, continuous renal replacement therapy; AKI, acute kidney injury; Cr, creatinine; Na, sodium; $\mathrm{K}$, potassium; $\mathrm{TCO}_{2}$, total carbon dioxide; $\mathrm{OR}$, odds ratio; $\mathrm{CI}$, confidence interval. a Adjusted for age, male, CKD, CHF, CCI, SAPS III, SOFA, urine output, $\mathrm{Cr}, \mathrm{Na}, \mathrm{K}, \mathrm{TCO}_{2}$, total bilirubin, hyperchloremia, and hypochloremia.
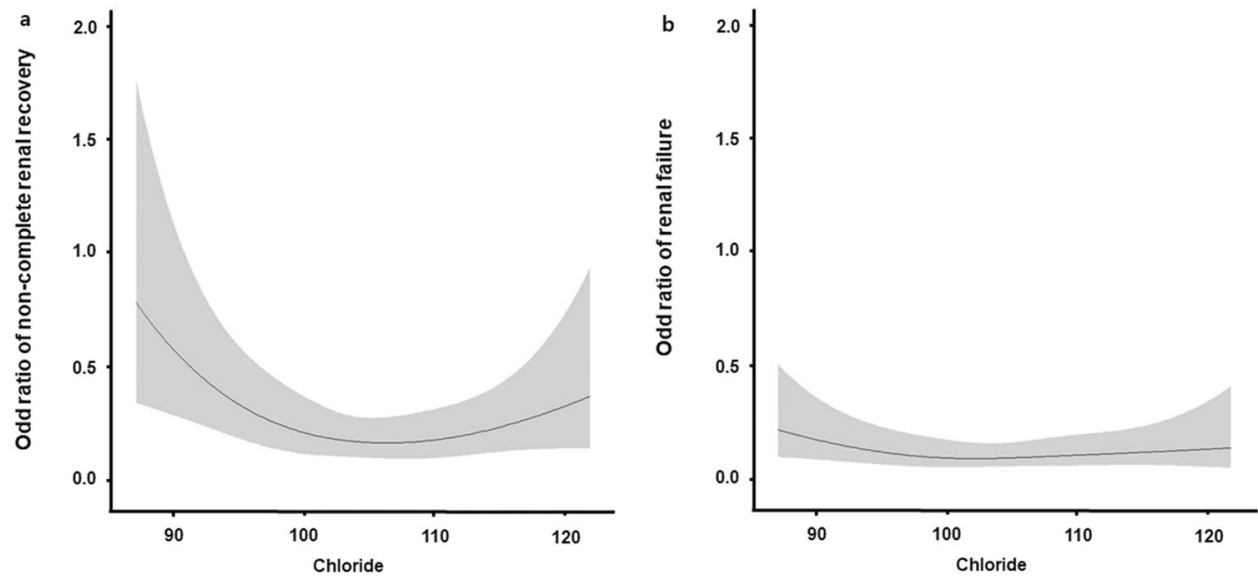

Figure 3. Restricted cubic spline plot of odds ratios for failure to restore renal function. Serum chloride concentrations have U-shaped associations with both (a) non-complete renal recovery and (b) renal failure. Multivariate logistic regression analysis adjusted for age, male, CKD, CHF, CCI, SAPS III, SOFA score, urine output, $\mathrm{Cr}, \mathrm{Na}, \mathrm{K}, \mathrm{TCO}_{2}$, total bilirubin, hypochloremia, and hyperchloremia. CKD, chronic kidney disease; CHF, congestive heart failure; CCI, Charlson comorbidity index; SAPS III, Simplified Acute Physiology Score III; SOFA, Sequential Organ Failure Assessment, Cr, creatinine; $\mathrm{Na}$, sodium; K, potassium; $\mathrm{TCO}_{2}$, total carbon dioxide. 
Severe AKI often requires renal replacement therapy in critically ill patients, which is known to be associated with a $20-50 \%$ mortality rate, development of CKD, and progression from CKD to renal failure ${ }^{24}$. Several epidemiologic studies showed that $10-30 \%$ of survivors with AKI requiring RRT remained dialysis-dependent ${ }^{24,25}$. In our study, the mortality rate (34.6\%) and incidence of renal failure (13.7\%) in patients with AKI requiring RRT were comparable to previous studies. In addition, electrolyte imbalances are very common clinical problems encountered in critically ill patients. Although the number of studies on chloride, known as forgotten anion, is limited, several studies have reported dyschloremia to also be very common (25-37\%) in critically ill patients. Similar to previous studies, dyschloremia was also observed in $28.6 \%$ of the current study population (hypochloremia $12.5 \%$ and hyperchloremia $16.1 \%)$.

Hypochloremia in critically ill patients can be caused by diuretic therapy, significant gastric drainage, vomiting, chronic respiratory acidosis, heart failure, syndrome of inappropriate antidiuretic hormone secretion, and excess infusion of hypotonic solutions ${ }^{26}$. Moreover, excessive chloride administration during resuscitation with chloride-rich solutions, osmotic diuresis, fever, hypermetabolic states, and post hypocapnia are conditions causing hyperchloremia in critically ill patient ${ }^{26}$. Therefore, dyschloremia could be a sign of severe illness. Furthermore, in the current study, SAPS III was higher in patients with dyschloremia than in those with normochloremia.

After AKI occurs, the kidney can recover from cellular damage. However, renal function may not be fully restored, with the development of CKD or progression from CKD to renal failure ${ }^{27}$. Because proximal tubular epithelial cells in renal tissue are not only located closest to the glomeruli but also have the highest metabolic rate, they are vulnerable to injury ${ }^{28}$. If the regenerative processes of these injured cells remain incomplete, these cells interact with other cell types in the interstitium, leading to renal fibrosis and eventually CKD or renal failure $^{28}$. Among patient-related factors in renal recovery following AKI, age, CKD, and comorbidity are the most frequently reported clinical factors ${ }^{29}$. Although illness severity scoring systems and organ dysfunction scores are thought to be additional clinical factors associated with renal recovery following $\mathrm{AKI}^{29}$, some studies have demonstrated that the prediction of renal recovery using these scoring systems was still ambiguous ${ }^{30,31}$. This study showed that high SAPS III and high SOFA scores were not associated with failure to restore renal function.

In this study, patients with dyschloremia had higher SAPS III than patients with normochloremia. However, dyschloremia was still independently associated with failure to restore renal function in survivors with severe AKI requiring RRT even after adjusting for other clinical factors such as SAPS III, suggesting that dyschloremia was associated with failure to restore renal function independent of the disease severity. Some studies found that hypochloremia was associated with increased intensive care units (ICU) length of stay or mortality ${ }^{22,32}$. Interestingly, in a study by Sho et al. ${ }^{22}$, compared with normochloremia, hypochloremia was an independent risk factor for the development of AKI. They hypothesized that the association of hypochloremia with AKI was mainly because of its association with potential hypovolemia, and not necessarily hypochloremia itself ${ }^{22}$. In contrast to the association of hypochloremia with renal function, the effects of hyperchloremia on renal function were well described. In vivo data suggested that vasoconstriction of the afferent arteriole of the renal glomerulus was induced by hyperchloremia ${ }^{17}$. In addition, an experimental study showed that renal blood flow and GFR were diminished after chloride infusion ${ }^{33}$. Furthermore, several clinical studies demonstrated that hyperchloremia was associated with the development of $\mathrm{AKI}^{19,20}$.

This study had some limitations. First, this was a small scale uncontrolled retrospective study. Therefore, there might be unmeasured confounding factors. Second, the study's design cannot elucidate whether dyschloremia has a causal role or is merely a consequence of certain conditions. Third, although there have been several studies exploring the effects of dyschloremia on renal function, to the best of our knowledge, there have not yet been any experimental studies explaining the role of chloride in the recovery of renal function following AKI. Fourth, because CRRT usually corrects dyschloremia within a few days, the effect of dyschloremia on renal function is disputable in this study.

In conclusion, despite the current study's limitations, it was the first to find an independent association of hypochloremia or hyperchloremia with failure to restore renal function following AKI. To clarify the role of dyschloremia in renal recovery following AKI, further well-designed clinical and experimental studies are needed.

\section{Methods}

Study design and patients. This observational retrospective study was conducted in 40-bed medical and surgical ICUs at university-affiliated hospitals from October 2009 to September 2019. Patients were eligible if they were aged 18 years or older, were admitted to the ICU, and received CRRT for severe AKI. In contrast, patients were excluded if they died or were referred to other clinics within 90 days after initiating CRRT, had pre-existing severe CKD (stage 4 or 5), had AKI caused by urinary tract obstruction, tumor lysis syndrome, thrombotic microangiopathy, or acute glomerulopathy, or received CRRT immediately after a kidney transplant. Of note, because treatments for tumor lysis syndrome, thrombotic microangiopathy, or acute glomerulopathy had direct effect on renal recovery, patients with these diseases were excluded. In addition, patients who did not have a record in the laboratory database within 12 months before hospital admission were also excluded.

Definitions. First, hypochloremia, normochloremia, and hyperchloremia were defined as serum chloride concentrations of $<96 \mathrm{mEq} / \mathrm{L}, 96-111 \mathrm{mEq} / \mathrm{L}$, and $>111 \mathrm{mEq} / \mathrm{L}$, respectively. If at least one of the following criteria was met, i.e., a urine output $<0.3 \mathrm{~mL} / \mathrm{kg} / \mathrm{h}$ for $\geq 24 \mathrm{~h}$, anuria for $\geq 12 \mathrm{~h}$, increase in serum creatinine concentration $\geq$ threefold from baseline, or serum creatinine concentration $\geq 4 \mathrm{mg} / \mathrm{dL}$ with an acute rise of at least $0.5 \mathrm{mg} / \mathrm{dL}$, a diagnosis of severe AKI was made. Second, baseline serum creatinine concentrations and estimated glomerular filtration rates (eGFR) were defined as average serum creatinine concentrations and eGFR within 
12 months before hospital admission, respectively. Non-complete renal recovery was defined as a $\geq 25 \%$ decline in baseline eGFR. Finally, renal failure was defined as a persistent dialysis-dependent state.

Continuous renal replacement therapy. The nephrologists in charge of the patients decided to initiate CRRT for patients with hyperkalemia (potassium level $>6.5 \mathrm{mEq} / \mathrm{L}$ ), metabolic acidosis $(\mathrm{pH}<7.15$ ), or diureticrefractory fluid overload (causing pulmonary edema). ICU nurses installed and maintained CRRT. In addition, the nephrologists considered stopping CRRT when the patients' serum creatinine concentration had decreased and if there was a return to a spontaneous urine output of $1000 \mathrm{~mL}$ per $24 \mathrm{~h}$. The femoral, internal jugular, or subclavian veins were utilized for CRRT. Although CRRT could not be discontinued, the patients' dialysis mode was changed to intermittent hemodialysis if the patients were hemodynamically stable. All patients received continuous veno-venous hemodiafiltration using Prismaflex machines (Baxter, IL, USA) or Multifiltrate machines (Fresenius Medical Care, Bad Homburg, Germany). CRRT was initially started with a blood flow rate of $100 \mathrm{~mL} /$ $\mathrm{min}$, which was gradually increased to $200 \mathrm{~mL} / \mathrm{min}$. The dialysate flow rate was $1000-2000 \mathrm{~mL} / \mathrm{h}$. The ultrafiltration dosage was set to at least $25 \mathrm{~mL} / \mathrm{kg} / \mathrm{h}$.

Data collection. The following data were collected from medical records at the time of CRRT; demographic data, causes of AKI requiring RRT, comorbidities, CCIs, clinical data such as SOFA scores and SAPS III, and biochemical data. Meanwhile, clinical data related to fluid balance, urine output, and chloride administration were collected between ICU admission and CRRT initiation. Cumulative fluid balance was defined as the difference between total intake and output during the period. Intake included fluids administered orally and parenterally, and output included urine, gastrointestinal losses, and drains. Total amount of chloride administration was calculated based on total intake. Cumulative fluid, total amount of chloride administrated, and total urine output were divided by the period. Furthermore, baseline serum creatinine concentrations and eGFR were collected as the average value for 12 months before hospital admission.

Outcome evaluation. The study objective was to evaluate the incidence of dyschloremia at CRRT initiation and determine its association with renal recovery at 90 days after initiating CRRT. Renal recovery was classified into non-complete renal recovery and renal failure.

Statistical analysis. Statistical analyses were performed using SPSS 19.0 (SPSS Inc., Chicago, Illinois, USA). Continuous variables are expressed as means \pm standard deviations or medians and inter-quartile ranges according to the normality of distribution. Categorical variables are summarized as numbers (percentages). Continuous variables were compared using Analysis of variance (AVONVA) or the Kruskal-Wallis test. Of note, Bonferonni post hoc tests or Dunn procedure was used for pairwise comparisons. Categorical variables were compared using chi-square test or Fisher's exact test. Logistic regression analysis was performed to assess variables associated with renal recovery. A multivariate analysis, which included all covariates with $P$ values of $<0.1$ in the univariate analysis, was performed. Although $P$-values were $\geq 0.1$, potential confounding factors were included in the multivariate analysis. To better represent the shape of the association between serum chloride concentrations and renal recovery, serum chloride concentrations were considered to be continuous variables, and odds ratios were modeled using a restricted cubic spline curve.

Ethical approval and informed consent. This study was performed in accordance with the Declaration of Helsinki and approved by the Institutional Review Board (IRB) of Kangdong Sacred Heart Hospital (Refs. Kangdong 2020-07-002). This was a retrospective medical record-based study, and the study subjects were deidentified. The IRB waived the need for written informed consent from the participants.

\section{Data availability}

The datasets generated during and/or analyzed during the current study are available from the corresponding author on reasonable request.

Received: 17 July 2020; Accepted: 23 October 2020

Published online: 12 November 2020

\section{References}

1. Morgera, S., Kraft, A. K., Siebert, G., Luft, F. C. \& Neumayer, H. H. Long-term outcomes in acute renal failure patients treated with continuous renal replacement therapies. Am. J. Kidney Dis. 40, 275-279 (2002).

2. Hsu, R. K., McCulloch, C. E., Dudley, R. A., Lo, L. J. \& Hsu, C. Y. Temporal changes in incidence of dialysis-requiring AKI. J. Am. Soc. Nephrol. 24, 37-42 (2013).

3. Cerda, J. et al. Epidemiology of acute kidney injury. Clin. J. Am. Soc. Nephrol. 3, 881-886 (2008).

4. Chawla, L. S. Acute kidney injury leading to chronic kidney disease and long-term outcomes of acute kidney injury: the best opportunity to mitigate acute kidney injury?. Contrib. Nephrol. 174, 182-190 (2011).

5. Brower, R. G. et al. Ventilation with lower tidal volumes as compared with traditional tidal volumes for acute lung injury and the acute respiratory distress syndrome. N. Engl. J. Med. 342, 1301-1308 (2000).

6. Chawla, L. S. et al. Association between AKI and long-term renal and cardiovascular outcomes in United States veterans. Clin. J. Am. Soc. Nephrol. 9, 448-456 (2014).

7. Berend, K., van Hulsteijn, L. H. \& Gans, R. O. Chloride: the queen of electrolytes?. Eur. J. Intern. Med. 23, 203-211 (2012).

8. Yunos, N. M., Bellomo, R., Story, D. \& Kellum, J. Bench-to-bedside review: chloride in critical illness. Crit. Care 14, 226 (2010).

9. Galla, J. H., Gifford, J. D., Luke, R. G. \& Rome, L. Adaptations to chloride-depletion alkalosis. Am. J. Physiol. 261, R771-781 (1991).

10. Kirchner, K. A., Kotchen, T. A., Galla, J. H. \& Luke, R. G. Importance of chloride for acute inhibition of renin by sodium chloride. Am. J. Physiol. 235, F444-450 (1978). 
11. Neyra, J. A. et al. Association of hyperchloremia with hospital mortality in critically Ill septic patients. Crit. Care Med. 43, 19381944 (2015).

12. Pfortmueller, C. A., Uehlinger, D., von Haehling, S. \& Schefold, J. C. Serum chloride levels in critical illness-the hidden story. Intensive Care Med. Exp. 6, 10 (2018).

13. Zazzeron, L. et al. Real-time urinary electrolyte monitoring after furosemide administration in surgical ICU patients with normal renal function. Ann. Intensive Care 6, 72 (2016).

14. Van Regenmortel, N., Verbrugghe, W., Van den Wyngaert, T. \& Jorens, P. G. Impact of chloride and strong ion difference on ICU and hospital mortality in a mixed intensive care population. Ann. Intensive Care 6, 91 (2016).

15. Boniatti, M. M., Cardoso, P. R., Castilho, R. K. \& Vieira, S. R. Is hyperchloremia associated with mortality in critically ill patients? A prospective cohort study. J. Crit. Care 26, 175-179 (2011).

16. Ter Maaten, J. M. et al. Hypochloremia, diuretic resistance, and outcome in patients with acute heart failure. Circ. Heart Fail. 9, e003109 (2016).

17. Wilcox, C. S. Regulation of renal blood flow by plasma chloride. J. Clin. Investig. 71, 726-735 (1983).

18. Lobo, D. N. \& Awad, S. Should chloride-rich crystalloids remain the mainstay of fluid resuscitation to prevent 'pre-renal' acute kidney injury?: con. Kidney Int. 86, 1096-1105 (2014).

19. Marttinen, M. et al. Association of plasma chloride values with acute kidney injury in the critically ill-a prospective observational study. Acta Anaesthesiol. Scand. 60, 790-799 (2016).

20. Zhang, Z., Xu, X., Fan, H., Li, D. \& Deng, H. Higher serum chloride concentrations are associated with acute kidney injury in unselected critically ill patients. BMC Nephrol. 14, 235 (2013).

21. Oh, H. J. et al. Baseline chloride levels are associated with the incidence of contrast-associated acute kidney injury. Sci. Rep. 7, 17431 (2017).

22. Shao, M. et al. Dyschloremia is a risk factor for the development of acute kidney injury in critically Ill patients. PLoS ONE 11, e0160322 (2016)

23. Rifkin, D. E., Coca, S. G. \& Kalantar-Zadeh, K. Does AKI truly lead to CKD?. J. Am. Soc. Nephrol. 23, 979-984 (2012).

24. Cerdá, J. et al. Promoting kidney function recovery in patients with AKI requiring RRT. Clin. J. Am. Soc. Nephrol. 10, 1859-1867 (2015).

25. Bagshaw, S. M. et al. Prognosis for long-term survival and renal recovery in critically ill patients with severe acute renal failure: a population-based study. Crit. Care 9, R700-709 (2005).

26. Bandak, G. \& Kashani, K. B. Chloride in intensive care units: a key electrolyte. F1000Res 6, 1930 (2017).

27. Bedford, M., Farmer, C., Levin, A., Ali, T. \& Stevens, P. Acute kidney injury and CKD: chicken or egg?. Am. J. Kidney Dis. 59, 485-491 (2012).

28. Qi, R. \& Yang, C. Renal tubular epithelial cells: the neglected mediator of tubulointerstitial fibrosis after injury. Cell Death Dis. 9, $1126(2018)$.

29. Forni, L. G. et al. Renal recovery after acute kidney injury. Intensive Care Med. 43, 855-866 (2017).

30. Basu, R. K., Chawla, L. S., Wheeler, D. S. \& Goldstein, S. L. Renal angina: an emerging paradigm to identify children at risk for acute kidney injury. Pediatr. Nephrol. 27, 1067-1078 (2012).

31. Uchino, S. et al. External validation of severity scoring systems for acute renal failure using a multinational database. Crit. Care Med. 33, 1961-1967 (2005).

32. Tani, M., Morimatsu, H., Takatsu, F. \& Morita, K. The incidence and prognostic value of hypochloremia in critically ill patients. ScientificWorldJournal 2012, 474185 (2012).

33. Chowdhury, A. H., Cox, E. F., Francis, S. T. \& Lobo, D. N. A randomized, controlled, double-blind crossover study on the effects of 2-L infusions of $0.9 \%$ saline and plasma-Lyte 148 on renal blood flow velocity and renal cortical tissue perfusion in healthy volunteers. Ann. Surg. 256, 18-24 (2012).

\title{
Acknowledgements
}

This work was supported by a National Research Foundation of Korea (NRF) Grant funded by the Korea government (Ministry of Science and ICT) (NRF-2017R1C1B5074168).

\section{Author contributions}

D.H.S. conceived and designed this study. Y.K.K., J.O., and H.J.J. acquired the data. D.H.S., J.O., and Y.K.K. analysed and interpreted the data. Y.K.K. wrote the paper. D.H.S., J.O., and H.J.J. reviewed the paper for important intellectual content and approved the final version.

\section{Competing interests}

The authors declare no competing interests.

\section{Additional information}

Correspondence and requests for materials should be addressed to D.H.S.

Reprints and permissions information is available at www.nature.com/reprints.

Publisher's note Springer Nature remains neutral with regard to jurisdictional claims in published maps and institutional affiliations.

\begin{abstract}
(c) (i) Open Access This article is licensed under a Creative Commons Attribution 4.0 International cc) License, which permits use, sharing, adaptation, distribution and reproduction in any medium or format, as long as you give appropriate credit to the original author(s) and the source, provide a link to the Creative Commons licence, and indicate if changes were made. The images or other third party material in this article are included in the article's Creative Commons licence, unless indicated otherwise in a credit line to the material. If material is not included in the article's Creative Commons licence and your intended use is not permitted by statutory regulation or exceeds the permitted use, you will need to obtain permission directly from the copyright holder. To view a copy of this licence, visit http://creativecommons.org/licenses/by/4.0/.
\end{abstract}

(C) The Author(s) 2020 